\title{
Possible multiorbital ground state in $\mathrm{CeCu}_{2} \mathrm{Si}_{2}$
}

\author{
Andrea Amorese, ${ }^{1}$ Andrea Marino, ${ }^{1,2}$ Martin Sundermann, ${ }^{3,1}$ Kai Chen, ${ }^{4}$ Zhiwei Hu, ${ }^{1}$ Thomas Willers, ${ }^{3, *}$ Fadi Choueikani, ${ }^{5}$ \\ Philippe Ohresser, ${ }^{5}$ Javier Herrero-Martin ${ }^{\circ},{ }^{6}$ Stefano Agrestini $\odot,{ }^{1,6, \dagger}$ Chien-Te Chen, ${ }^{7}$ Hong-Ji Lin, ${ }^{7}$ Maurits W. Haverkort, ${ }^{8}$ \\ Silvia Seiro $₫,{ }^{1}, 末$ Christoph Geibel, ${ }^{1}$ Frank Steglich, ${ }^{1}$ Liu Hao Tjeng ${ }^{\circ},{ }^{1}$ Gertrud Zwicknagl, ${ }^{9}$ and Andrea Severing $\oplus^{3,1}$ \\ ${ }^{1}$ Max Planck Institute for Chemical Physics of Solids, Nöthnitzer Straße 40, 01187 Dresden, Germany \\ ${ }^{2}$ Dipartimento di Fisica, Politecnico di Milano, Piazza Leonardo da Vinci 32, I-20133 Milano, Italy \\ ${ }^{3}$ Institute of Physics II, University of Cologne, Zülpicher Straße 77, 50937 Cologne, Germany \\ ${ }^{4}$ Helmholtz-Zentrum Berlin für Materialien und Energie, Albert-Einstein-Straße 15, 12489 Berlin, Germany \\ ${ }^{5}$ Synchrotron SOLEIL, L'Orme des Merisiers, Saint-Aubin, 91192 Gif-sur-Yvette, France \\ ${ }^{6}$ ALBA Synchrotron Light Source, E-08290 Cerdanyola del Vallès, Barcelona, Spain \\ ${ }^{7}$ National Synchrotron Radiation Research Center (NSRRC), Hsinchu 30076, Taiwan \\ ${ }^{8}$ Institute for Theoretical Physics, Heidelberg University, Philosophenweg 19, 69120 Heidelberg, Germany \\ ${ }^{9}$ Institute for Mathematical Physics, Technische Universität Braunschweig, D-38106 Braunschweig, Germany
}

(Received 3 October 2020; revised 7 December 2020; accepted 8 December 2020; published 28 December 2020; corrected 12 February 2021)

\begin{abstract}
The crystal-field ground-state wave function of $\mathrm{CeCu}_{2} \mathrm{Si}_{2}$ has been investigated with linearly polarized $M$ edge x-ray absorption spectroscopy from $250 \mathrm{mK}$ to $250 \mathrm{~K}$, thus covering the superconducting $\left(T_{\mathrm{c}}=0.6 \mathrm{~K}\right)$, Kondo $\left(T_{\mathrm{K}} \approx 20 \mathrm{~K}\right)$, and Curie-Weiss regimes. The comparison with full multiplet calculations shows that the temperature dependence of the experimental linear dichroism is well explained with a $\Gamma_{7}^{(1)}$ crystal-field ground state and the thermal population of excited states at around $30 \mathrm{meV}$. The crystal-field scheme does not change throughout the entire temperature range thus making the scenario of orbital switching unlikely. Spectroscopic evidence for the presence of the $\mathrm{Ce} 4 f^{0}$ configuration in the ground state is consistent with the possibility for a multiorbital character of the ground state. We estimate from the Kondo temperature and crystal-field splitting energies that several percents of the higher lying $\Gamma_{6}$ state and $\Gamma_{7}^{(2)}$ crystal-field states are mixed into the primarily $\Gamma_{7}^{(1)}$ ground state. This estimate is also supported by renormalized band-structure calculations that uses the experimentally determined crystal-field scheme.
\end{abstract}

DOI: $10.1103 /$ PhysRevB.102.245146

\section{INTRODUCTION}

Heavy fermion compounds are $f$ electron systems where, at low temperatures, the hybridization of localized $4 f$ or $5 f$ and conduction electrons ( $c f$ hybridization) forms an entangled ground state with quasiparticles that can have effective masses up to three orders of magnitude larger than the free electron mass [1,2]. The $c f$ hybridization goes along with a certain delocalization of the $f$ electrons and depending on the degree of delocalization magnetic order, unconventional superconductivity, or intermediate valence occurs. Here superconductivity usually occurs in the vicinity of the quantum

\footnotetext{
*Present address: Krüss GmbH, Borsteler Chaussee 85, 22453 Hamburg, Germany.

${ }^{\dagger}$ Present address: Diamond Light Source, Harwell Science and Innovation Campus, Didcot, OX11 0DE, United Kingdom.

${ }^{\ddagger}$ Present address: Leibniz IFW Dresden, Helmholtzstraße 20, 01069 Dresden, Germany.
}

Published by the American Physical Society under the terms of the Creative Commons Attribution 4.0 International license. Further distribution of this work must maintain attribution to the author(s) and the published article's title, journal citation, and DOI. Open access publication funded by the Max Planck Society. critical point where the magnetic order transitions are suppressed to zero Kelvin $[3,4]$. In the heavy fermion compound $\mathrm{CeCu}_{2} \mathrm{Si}_{2}$, a material with a Kondo temperature of $T_{\mathrm{K}} \approx$ $20 \mathrm{~K}[5,6]$, unconventional superconductivity was observed [7], opening up an entire field of research. Superconductivity in $\mathrm{CeCu}_{2} \mathrm{Si}_{2}$ appears at ambient pressure but also in a wider range of applied pressures where two superconducting domes have been observed with maxima at $0.45 \mathrm{GPa}\left(T_{\mathrm{c}}=0.6 \mathrm{~K}\right)$ and $4.5 \mathrm{GPa}\left(T_{\mathrm{c}}=2 \mathrm{~K}\right)$ in the pressure $(P) /$ temperature $(T)$ phase diagram [8]. The substitution of Si by the larger Ge separates the two domes [9], suggesting the two superconducting phases may be of different origin.

The ambient or low-pressure superconducting phase is close to antiferromagnetism; small changes in the $\mathrm{Si}$ stoichiometry lead to an antiferromagnetic ground state [10]. It is therefore likely that spin fluctuations are responsible for the formation of Cooper pairs and there is strong evidence for the $d$-wave character of this superconducting phase [11-13]. For the high-pressure superconducting phase, however, valence fluctuations were proposed to provide the pairing mechanism [14], but so far a valence transition at applied pressure has not been experimentally confirmed [15].

The $d$-wave character of the ambient pressure superconductivity in $\mathrm{CeCu}_{2} \mathrm{Si}_{2}$ has been contested recently [16-18] and here the determination of the crystal-field wave functions 

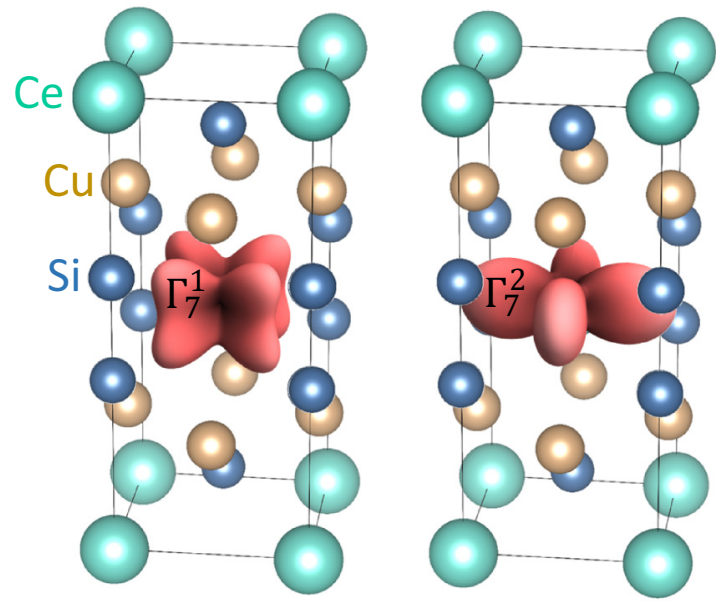

FIG. 1. $\mathrm{ThCr}_{2} \mathrm{Si}_{2}$ structure of $\mathrm{CeCu}_{2} \mathrm{Si}_{2}$ with $\mathrm{Ce} \Gamma_{7}^{(1)}$ (left) and $\Gamma_{7}^{(2)}$ orbital (right) at the body center of the unit cell. The aspect ratios of the orbitals correspond to Eqs. (1) and (2) with $\alpha=0.59$ and the orientation to $\alpha<0$.

of the crystal-field split Hund's rule ground state has become important. Is the ground state a multiorbital state as suggested by Ref. [19]? Or does an orbital switching as function of temperature take place, as suggested in another scenario to model the double-dome structure of the two superconducting phases [20]? For further clarification, it is therefore indispensable to revisit spectroscopically the crystal-field problem of $\mathrm{CeCu}_{2} \mathrm{Si}_{2}$ and its temperature dependence.

We recall the $J=5 / 2$ Hund's rule ground state of $\mathrm{Ce}$ in $\mathrm{CeCu}_{2} \mathrm{Si}_{2}$ is split by the tetragonal crystal field into two $\Gamma_{7}^{(1,2)}$ and one $\Gamma_{6}$ Kramers doublet, which can be written in $J_{z}$ representation with $0 \leqslant|\alpha| \leqslant 1$ :

$$
\begin{gathered}
\Gamma_{7}^{(1)}=\alpha| \pm 5 / 2\rangle+\sqrt{\left(1-\alpha^{2}\right)}|\mp 3 / 2\rangle, \\
\Gamma_{7}^{(2)}=\sqrt{\left(1-\alpha^{2}\right)}| \pm 5 / 2\rangle-\alpha|\mp 3 / 2\rangle, \\
\Gamma_{6}=| \pm 1 / 2\rangle .
\end{gathered}
$$

Here the $\Gamma_{7}^{(1,2)}$ orbital states distinguish each other in their $J_{z}$ admixture and orientation within the unit cell: For $\alpha>0$, the lobes of the angular distribution of the $\Gamma_{7}^{(1)}$ state points along [100] and for $\alpha<0$ along [110] (see Fig. 1), whereas for $\alpha=0$, i.e., in the case of a pure $J_{z}$ state, the orbital has full rotational symmetry around the [001] axis. Goremychkin et al. found with inelastic neutron scattering two strongly broadened and almost degenerate crystal-field excitations at about $30 \mathrm{meV}$ [21]. In the Goremychkin neutron experiment, the crystal-field wave functions were, however, determined from the anisotropy of the static susceptibility $\chi_{\text {stat }}$, which is well described with a $\Gamma_{7}^{(1)}$ ground state with $|\alpha|=0.88$. Note that inelastic neutron scattering cannot determine the sign of $\alpha$ in the wave function since it is dipole limited. Nonresonant inelastic x-ray scattering (NIXS) overcomes this dipole limitation [22-24] and Willers et al. found that $\alpha$ in $\Gamma_{7}^{(1)}$ is negative at $20 \mathrm{~K}$; i.e., the $\Gamma_{7}^{(1)}$ with its lobes along the (110) direction forms the ground state at $20 \mathrm{~K}$ [25]. The NIXS experiment was performed well above $T_{\mathrm{K}}$ and $T_{\mathrm{c}}$ without looking explicitly at the $J_{z}$ admixture ( $a c$ anisotropy). Hence, there has been no spectroscopic information available about the $J_{z}$ admixture of the ground-state wave function of $\mathrm{CeCu}_{2} \mathrm{Si}_{2}$ or about the possibility of a $c f$-hybridization induced multiorbital ground state [19] or orbital reoccupation [20].

The present work addresses this lack of information. We set up an experiment with the aim to investigate the crystalfield wave functions of the ground state, below $T_{\mathrm{K}}$ and $T_{\mathrm{c}}$, and well above, looking also for any changes in the orbital occupation that cannot be explained with the Boltzman-type thermal occupation of excited crystal-field states.

\section{METHOD}

$\mathrm{X}$-ray absorption spectroscopy (XAS) is an element specific probe for valence, spin, and orbital degrees of freedom [26-28]. In particular, the linear dichroism of linearly polarized XAS (XLD) at the rare-earth $M_{4,5}$ edges $\left(3 d^{10} 4 f^{1}\right.$ $\rightarrow 3 d^{9} 4 f^{2}$ ) measures the spatial anisotropy of the ionic $4 f^{1}$ crystal-field split ground state with unprecedented accuracy [29-33]. XAS is element specific and the signal to background ratio is very good. The linear dichroism probes specifically the ground-state symmetry when working at low $T$. Excited crystal-field states contribute via thermal occupation.

The linear dichroism method is based on dipole selection rules and each $J_{z}$ state exhibits its own specific directional dependence [29], resulting in specific dichroisms $\mathrm{D}_{J_{2}}$ that relate to each other as $\mathrm{D}_{5 / 2}=-5 \mathrm{D}_{3 / 2}=-1.25 \mathrm{D}_{1 / 2}$. Although the data analysis was performed with a full multiplet calculation (see below), the analysis becomes more intuitive when expressing the linear dichroism of each crystal-field state in terms of incoherent sums of the individual $\mathrm{D}_{J_{z}}$. This is only possible when the rotational symmetry is higher than twofold and as long as the crystal-field splitting is small with respect to the spin orbit splitting [29]. Both are fulfilled for $\mathrm{CeCu}_{2} \mathrm{Si}_{2}$ so that we can write for the linear dichroism of the $\Gamma_{7}^{(1)}$ state:

$$
D_{\Gamma_{7}^{(1)}}=\alpha^{2} D_{5 / 2}+\left(1-\alpha^{2}\right) D_{3 / 2} .
$$

The linear dichroism $D_{\Gamma_{7}^{2}}$ of the $\Gamma_{7}^{(2)}$ state can be written accordingly. This little exercise demonstrates that the linear dichroism is sensitive to the square of $\alpha$ and therefore not to its sign. When excited states get populated with rising temperature, the total linear dichroism) signal is the superposition of the individual linear dichroisms of each crystal-field state, weighted by thermal occupation.

A proof for the existence of $c f$ hybridization is the presence of a satellite in the $M_{4,5}$-edge spectra. It arises from the $c f$-hybridization induced mixture of the $4 f^{1}\left(\mathrm{Ce}^{3+} J=5 / 2\right)$ and $\left.4 f^{0} \mathrm{Ce}^{4+} J=0\right)$ configurations. The core hole splits up the final states that can be reached from the quantum mechanical entangled ground state given by $\sqrt{n_{1}} f^{1}+\sqrt{n_{0}}$ $f^{0}$ [34] since it acts differently on different configurations. Here $n_{1}$ and $n_{0}$ label the weights of the two configurations. The satellite indicating the presence of $4 f^{0}$ in the ground state shows up at the high-energy tail of the $4 f^{1}$ absorption lines; here we are always referring to the configurations of the initial state [29]. These satellites can be used for measuring relative changes of the $4 f$ shell occupation with temperature [31]. 


\section{EXPERIMENT AND SIMULATIONS}

The XAS experiment was performed on well-characterized superconducting $\mathrm{CeCu}_{2} \mathrm{Si}_{2}$ single crystals [35] at the DEIMOS beamline at synchrotron SOLEIL in France [36] between 0.25 and $5 \mathrm{~K}$, at the BOREAS beamline at synchrotron ALBA in Spain [37] between 3.2 and $150 \mathrm{~K}$, and at the DRAGON beamline of the NSRRC in Taiwan between 100 and $250 \mathrm{~K}$. The energy resolution at the Ce $M_{4,5}$ edge at $h v \approx 870-910 \mathrm{eV}$ was about $0.4 \mathrm{eV}$. The DEIMOS beamline is quite unique in the world since its cryomagnet is equipped with an insert for cooling to $250 \mathrm{mK}$ in ultrahigh vacuum (UHV) [38]. The temperature stability is within a few percent and the temperature difference between thermocouple and sample surface amounts to 50 to $100 \mathrm{mK}$, depending on thermal contact. The BOREAS beamline has the advantage of a reference sample in the beam so that small drifts in energy can be easily corrected. At all beamlines, the samples were cleaved in situ in ultrahigh vacuum and then transferred to a main chamber $\left(10^{-10}\right.$ mbar), where the signal was recorded in the total electron yield (TEY) mode by measuring the drain current. The cleaved $a c$ surface was perpendicular to the Poynting vector, with $c$ being the fourfold tetragonal axis, so that data could be taken with the electric field vector $\vec{E} \| c$ and $\vec{E} \perp c$. This was achieved at the DEIMOS and BOREAS beamlines by changing the polarization of the light impinging on the sample. At the DRAGON beamline, the polarization cannot be changed and instead the sample was turned to achieve the polarization parallel and perpendicular to the $c$ axis. All samples were aligned with the Laue method prior to the experiment.

The data were normalized to the integrated intensity of the experimental isotropic spectra, constructed as $I_{\text {iso }}=\left(I_{\vec{E} \| c}+\right.$ $\left.2 I_{\vec{E} \perp c}\right) / 3$ and compared with simulations obtained with the full multiplet code QUANTY [39]. The atomic parameters for the $4 f-4 f$ and $3 d-4 f$ Coulomb interactions were calculated with the COWAN code [40] and reduced by about $21 \%$ and $39 \%$, respectively, to account for configuration interaction effects that are not included in the Hartee-Fock scheme. The reduction factors are determined by optimizing the XAS simulation to $I_{\text {iso }}$ without taking into account the crystal-field splitting of the Hund's rule ground state. Configuration interaction effects are not considered. For obtaining the best crystal-field description of the linear polarized data, the linear dicroism $I_{\vec{E} \| c}-I_{\vec{E} \perp c}$ was fitted because the background, due to the edge jump, and the $4 f^{0}$ satellite do not show any dichroism and so cancel out in the linear dichroism.

\section{RESULTS}

Figure 2(a) shows the XAS data of $\mathrm{CeCu}_{2} \mathrm{Si}_{2}$ at $0.25 \mathrm{~K}$ and Fig. 2(b) shows the simulation based on a single ion crystal-field model with a ground state $\Gamma_{7}^{(1)}$ [Eq. (1)] with $\alpha=|0.59| \pm 0.1$. The comparison of the experimental and simulated dichroism $I_{\vec{E} \| c}-I_{\vec{E} \perp c}$ in Fig. 2(c) establishes how well the data are reproduced.

The temperature dependence of the linear dichroism is displayed in Figs. 3(a)-3(d): for $T=0.25$ to $5 \mathrm{~K}$ in Figs. 3(a) and 3(b), for $T=3.2$ to $150 \mathrm{~K}$ in Figs. 3(c) and 3(d), and for $T=100$ to $250 \mathrm{~K}$ in Figs. 3(e) and 3(f). Within the accuracy
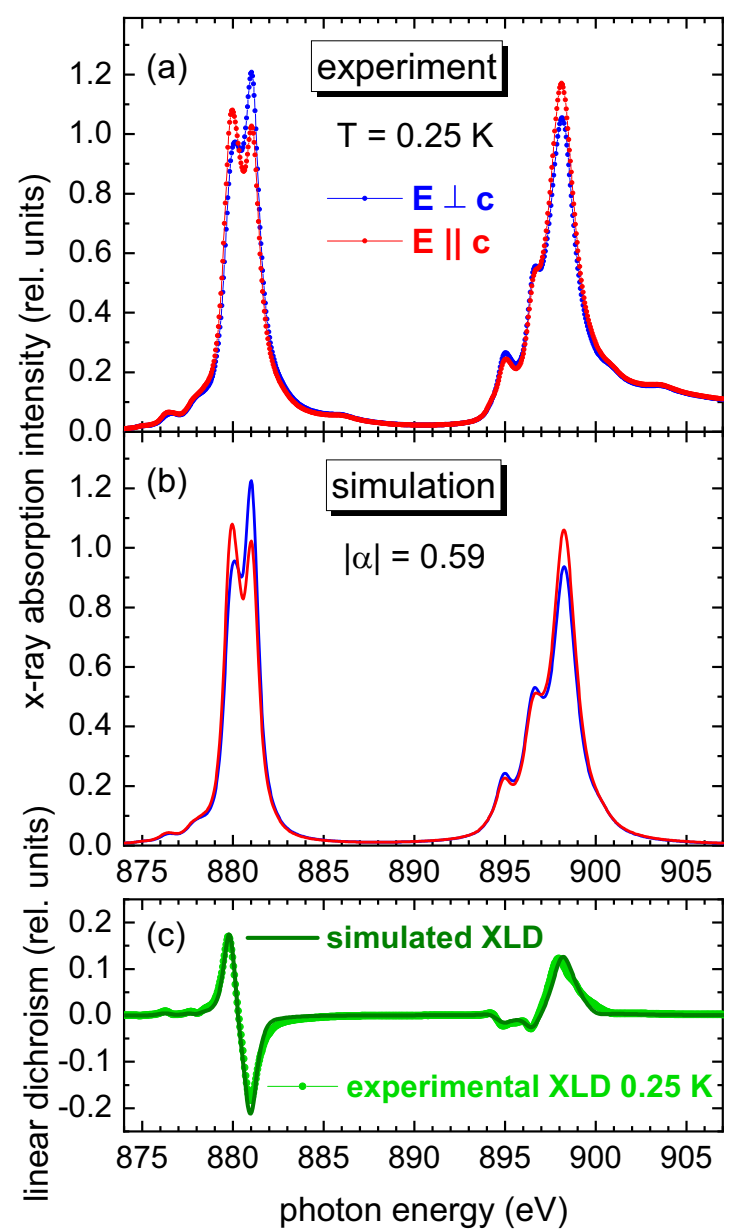

FIG. 2. (a) Experimental linear polarized x-ray absorption data $I_{\vec{E} \| c}$ and $I_{\vec{E} \perp c}$ of $\mathrm{CeCu}_{2} \mathrm{SI}_{2}$ at $250 \mathrm{mK}$, (b) simulation with a $\Gamma_{7}^{(1)}$ ground-state wave function [see Eq. (1)] and $|\alpha|=0.59$, and (c) the linear dichroism $I_{\vec{E} \| c}-I_{\vec{E} \perp c}$ (XLD), demonstrating the excellent agreement between data (light green dots) and simulation (dark green line).

of the experiment, there is no change in the linear dichroism up to $75 \mathrm{~K}$. At $150 \mathrm{~K}$, however, the linear dichroism decreases a fair amount. Figures $3(\mathrm{~g})$ and $3(\mathrm{~h})$ show the simulated linear dichroism on the basis of a $\Gamma_{7}^{(1)}$ ground-state wave function with $\alpha=|0.59|$ and excited states $\Gamma_{6}$ and $\Gamma_{7}^{(2)}$ at around $30 \mathrm{meV}$ : The latter crystal-field splitting energies are from the neutron results in Ref. [21]. The simulations reproduce the experimental data very well. A crystal-field state at, e.g., $12 \mathrm{meV}$ as suggested by Horn et al. [41], on the other hand, would lead to a much faster reduction of linear dichroism and can therefore be excluded.

\section{DISCUSSION}

The XAS spectra and the linear dichroism therein of In $\mathrm{CeCu}_{2} \mathrm{Si}_{2}$ have been measured in the wide temperature range from $250 \mathrm{mK}$ to $250 \mathrm{~K}$. The data are all well described with the single-orbital crystal-field ground-state wave function

$$
\Gamma_{7}^{(1)}=-0.59| \pm 5 / 2\rangle+0.81|\mp 3 / 2\rangle
$$




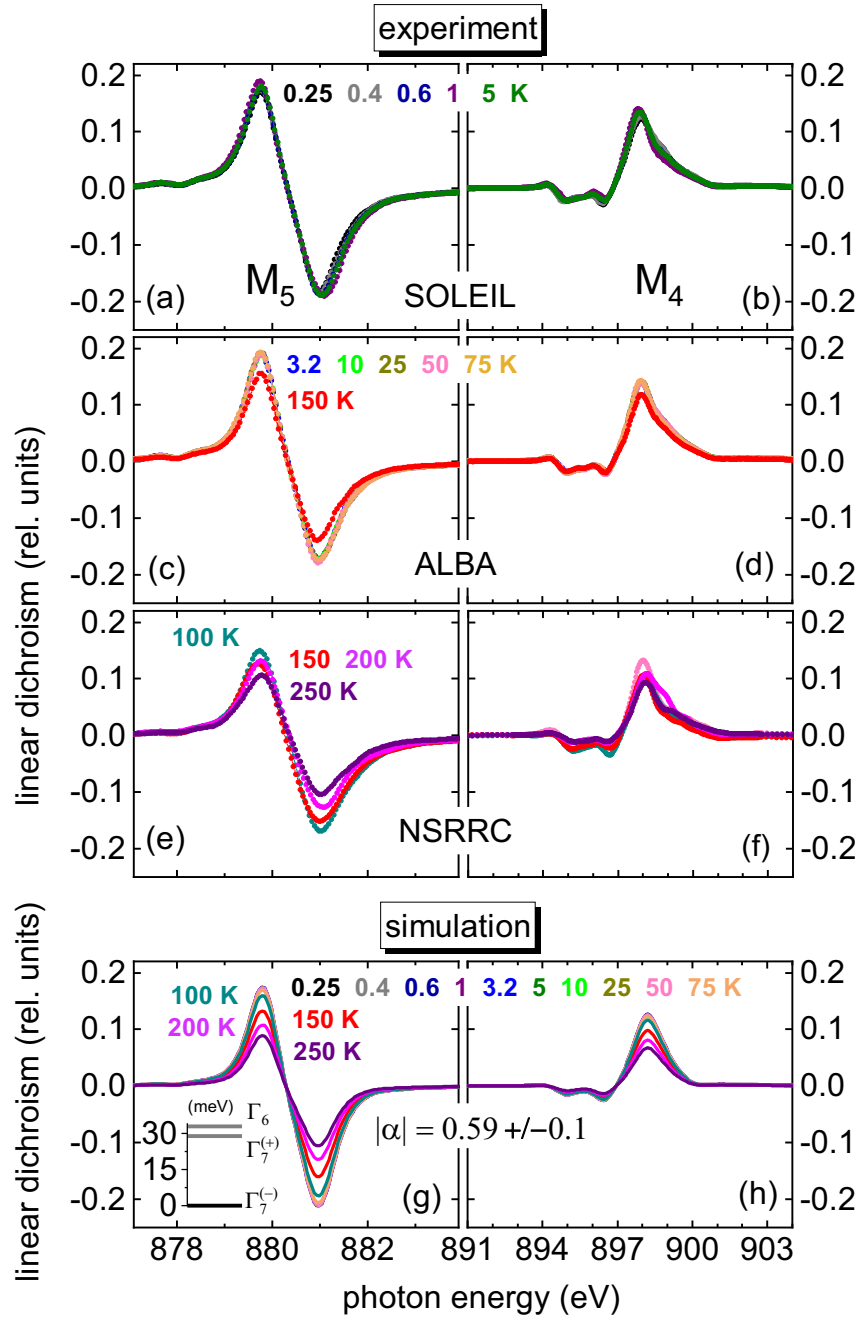

FIG. 3. Experimental linear dichroism $I_{\vec{E} \| c}-I_{\vec{E} \perp c}$ at the $M_{5}$ and $M_{4}$ edges for $T=0.25$ to $5 \mathrm{~K}$ (SOLEIL data) in panels (a) and (b), for $T=3.2$ to $150 \mathrm{~K}$ (ALBA data) in panels (c) and (d), and for $T=100$ to $250 \mathrm{~K}$ (NSRRC data) in panels (e) and (f). Panels (g) and (h) show the full multiplet simulations taking into account the thermal population of crystal-field states at around $30 \mathrm{meV}$.

and the Boltzman occupation of the excited states at $\approx 30 \mathrm{meV}$. We especially note that there are no noticeable spectral changes while going from the superconducting, via the Kondo, to the Curie-Weiss regime up to $75 \mathrm{~K}$. This strongly suggest that the orbital switching as function of temperature as suggested in Ref. [20] does not take place. Instead, the XAS results point toward a robust static crystal-field scheme. The orbital switching scenario was also discarded for $\mathrm{CeCu}_{2} \mathrm{Ge}_{2}$ where the in-plane anisotropy of the occupied orbital was measured as function of temperature. In the case of an orbital reoccupation of the $\Gamma_{7}^{(1)}$ and $\Gamma_{7}^{(2)}$ states, the directional dependence of the NIXS signal should have flipped. This, however, was not observed [42].

The question is now whether the ground state is given by just one crystal-field state or whether higher lying crystal-field states contribute. The answer to this question is of relevance for the development of multiorbital-based models [19] that perhaps could explain why the $d$-wave superconductor
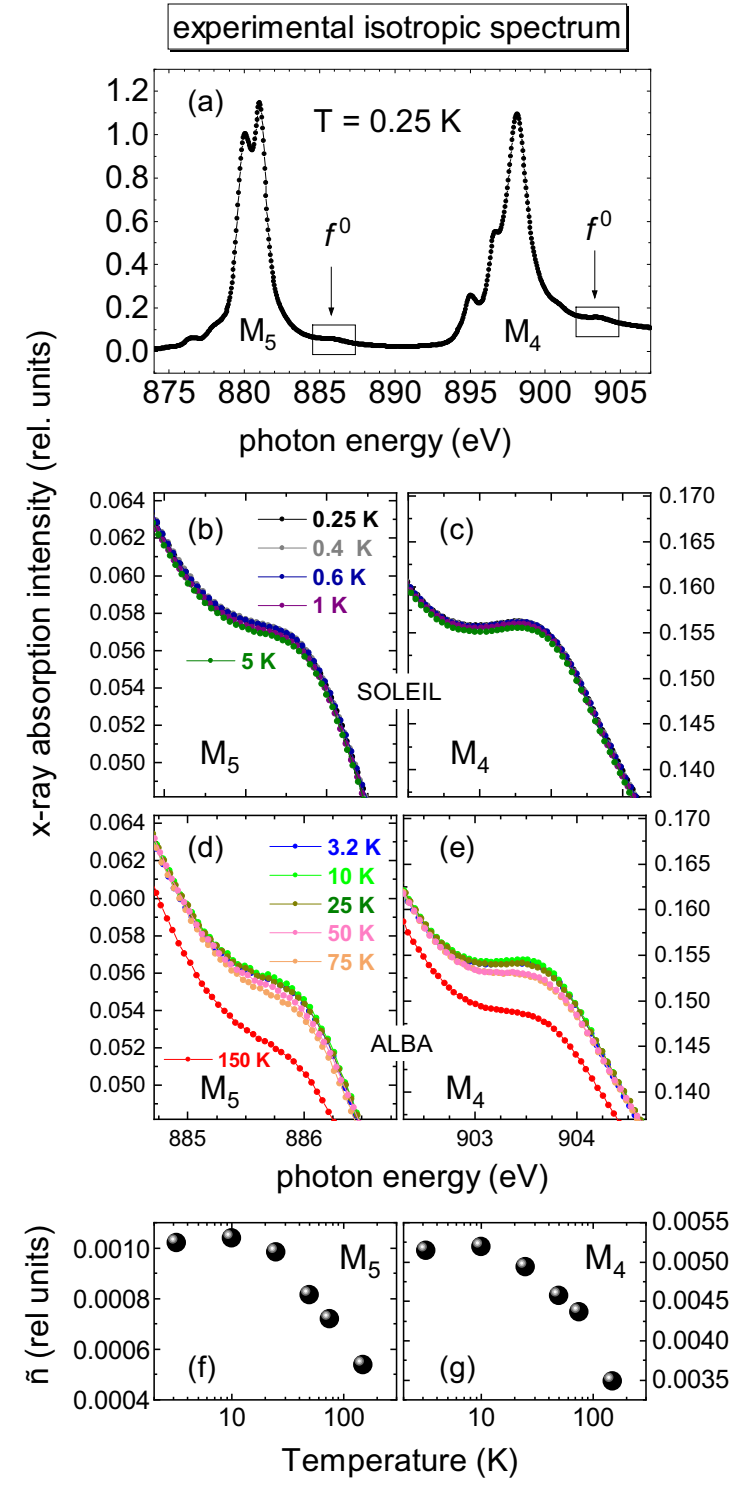

FIG. 4. (a) Isotropic XAS data $I_{\text {iso }}=\left(I_{\vec{E} \| c}+2 I_{\vec{E} \perp c}\right) / 3$ of $\mathrm{CeCu}_{2} \mathrm{Si}_{2}$ at $0.25 \mathrm{~K}$. [(b)-(e)] $f^{0}$ satellite on an enlarged scale [see rectangles in panels (a) and (b)] for 0.25 to $5 \mathrm{~K}$ (SOLEIL data) in panels (a) and (b) and for 3.2 to $150 \mathrm{~K}$ (ALBA data) in panels (d) and (e). [(f), (g)] Integrated intensity $\tilde{n}$ of $f^{0}$ satellite in panels (d) and (e) after subtracting a linear background.

$\mathrm{CeCu}_{2} \mathrm{Si}_{2}$ may be fully gaped at sufficiently low temperatures [16-18].

The experimental XAS spectra, see Fig. 4(a), exhibit the small but distinct feature at about $886 \mathrm{eV}$ photon energy, which can be attributed to the presence of the Ce $4 f^{0}$ configuration in the ground state [34]. This in turn signals that the $c f$ hybridization is active and thus also provides a channel for the higher lying crystal-field states to mix into the ground state. The amount of mixing is not negligible for systems with high Kondo temperatures such as $\mathrm{CeRu}_{4} \mathrm{Sn}_{6}$ [43] and also $\mathrm{CeCoIn}_{5}$ [44], where the crystal-field splittings are not much larger than the Kondo energy scale. For $\mathrm{CeCu}_{2} \mathrm{Si}_{2}$, however, we expect that the involvement of the higher crystal-field states will be 
quite small because transport and thermodynamic measurements indicate a Kondo temperature of about $20 \mathrm{~K}[5,6]$, which is small with respect to the crystal-field splitting $\Delta_{\Gamma} \approx 30 \mathrm{meV}$ [21].

In the following, we will utilize our spectroscopy to obtain an independent check on the Kondo energy scale by looking at the evolution of the relative $4 f^{0}$ spectral weight as function of temperature. Figures 4(b)-4(e) display the $f^{0}$ satellites [see rectangles in Figs. 4(a) and 4(b)] on an enlarged scale. We find that the $4 f^{0}$ spectral weight remains unchanged up to 5 K [Figs. 4(b) and 4(c)] within the accuracy of the data, decreases slightly at 50 and $75 \mathrm{~K}$ [Figs. 4(d) and 4(e)], and is strongly reduced at $150 \mathrm{~K}$. Figures $4(\mathrm{f})$ and $4(\mathrm{~g})$ show $\tilde{n}(T)$, the integrated intensity of the $f^{0}$ satellite between $T=3.2$ and $150 \mathrm{~K}$ after subtracting a linear background. According to a self-consistent large-orbital degeneracy theory by Bickers et al. [45], the presence of the Kondo effect is reflected in the temperature dependence of $\tilde{n}(T)$. It should show a gradual decrease with temperature followed by a flattening out for $T=\infty \gg T_{\mathrm{K}}$. In particular, at $T_{\mathrm{K}}$ it exhibits an inflection point at $1 / 2$ of the difference of its high- and low-temperature value. This scenario, however, does not take crystal-field effects into account. Therefore, although here we do not observe the inflection point nor flattening out of $\tilde{n}(T)$, possibly because of the population of excited crystal-field states (see Ref. [46]), we do observe that $\tilde{n}(T)$ starts to drop for $T>20 \mathrm{~K}$. We take this as a sign for coming out of the low-temperature regime where the Kondo interaction is most effective and therefore interpret the $\tilde{n}(T)$ data in terms of a Kondo temperature of about $20 \mathrm{~K}$. This value is in good agreement with the the literature value of $T_{\mathrm{K}}=20 \mathrm{~K}[5,6]$. We thus find $\mathrm{CeCu}_{2} \mathrm{Si}_{2}$ is indeed a material with a relatively low $T_{\mathrm{K}}$.

We now carry out an analysis using a simple approximation scheme for the Anderson impurity Hamiltonian [47] in order to estimate how much of the higher lying $\Gamma_{6}$ and $\Gamma_{7}^{(2)}$ crystalfield states contribute to the primarily $\Gamma_{7}^{(1)}$ ground state. The approximate description is valid at temperatures $T \lesssim T_{0}$, where $T_{0}$ is the characteristic temperature of heavy fermion or valence fluctuating systems and the results smoothly reduce to the predictions of the variational treatment [34,48]. Assuming a constant density of conduction states $N(0)$, the occupancies of the crystal-field split $4 f$ states are given by

$$
n_{f \Gamma \tau} \sim\left(1-n_{f}\right)\left|V_{\Gamma}\right|^{2} N(0) \frac{1}{k_{B} T_{0}+\Delta_{\Gamma}},
$$

where $\Gamma$ refers to the representation, i.e., $\Gamma=\Gamma_{7}^{(1)}, \Gamma_{6}$, or $\Gamma_{7}^{(2)}, \tau= \pm$ accounts for the Kramers degeneracy, and $\Delta_{\Gamma}$ is the crystal-field excitation energy relative to the crystal-field ground state. This expression is a straightforward generalization of the formula given in Ref. [34]. In the systems under consideration, the crystal-field splitting largely exceeds the characteristic energy $\Delta_{\Gamma} \gg k_{B} T_{0}$. The $c f$ hybridization $\left|V_{\Gamma}\right|$ may depend on the symmetry of the crystal-field state $\Gamma$ and the weight of the $f^{0}$ configuration in the ground state is given by

$$
\left(1-n_{f}\right)=\frac{1}{1+\sum_{\Gamma^{\prime}} \frac{2\left|V_{\Gamma^{\prime}}\right|^{2} N(0)}{k_{B} T_{0}+\Delta_{\Gamma^{\prime}}}}
$$
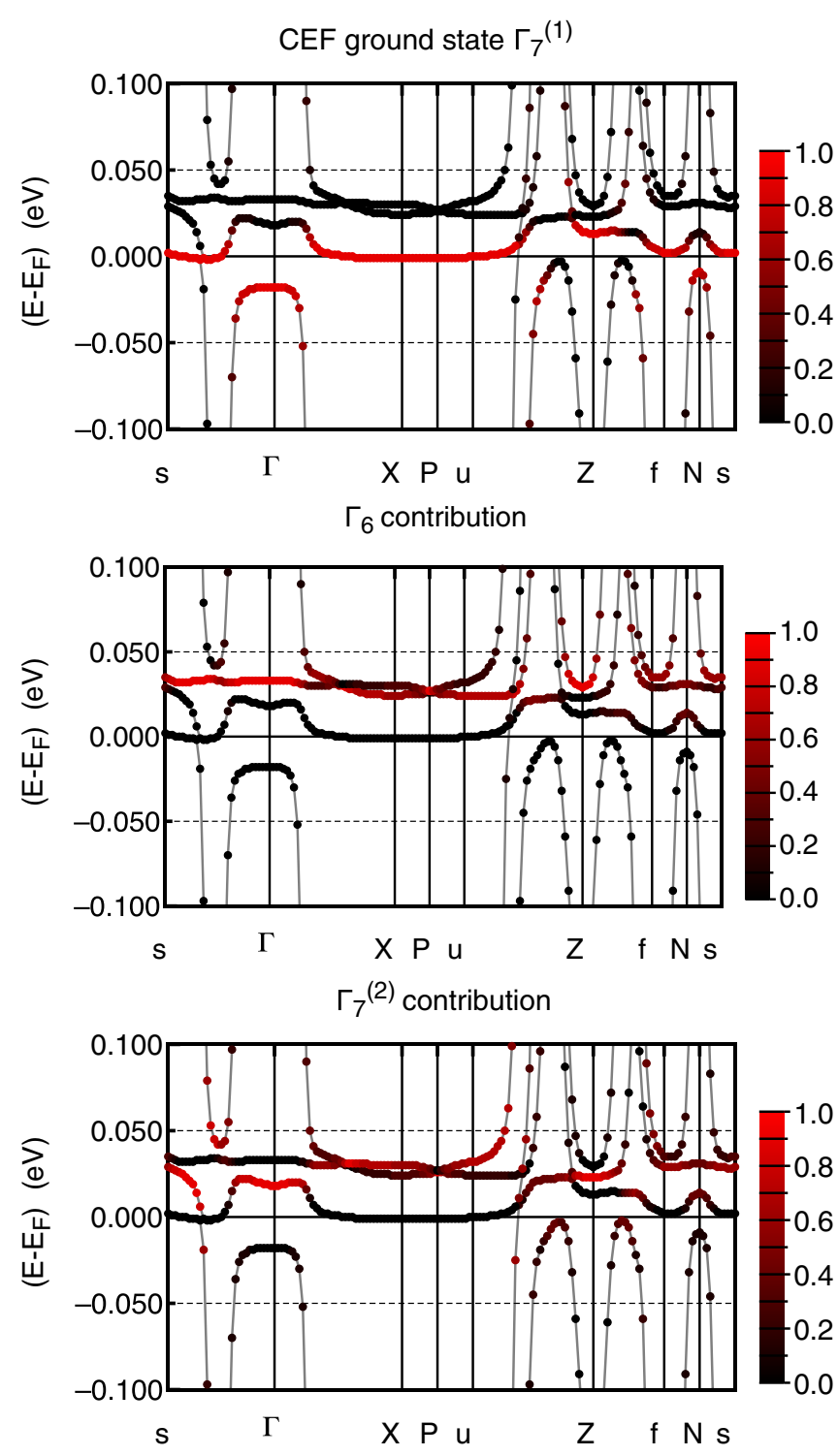

FIG. 5. Renormalized quasiparticle bands, showing the contributions of each crystal-field state.

In $\mathrm{CeCu}_{2} \mathrm{Si}_{2},\left(1-n_{f}\right) \approx 0.01-0.03$ and the dominant contribution to the low-energy states comes from the $\Gamma_{7}^{1}$ crystal-field ground state. We anticipate, however, a $\Gamma_{6}$ contribution

$$
\sum_{\tau= \pm} n_{f \Gamma_{6} \tau}=\frac{\frac{2\left|V_{\Gamma_{6}}\right|^{2} N(0)}{k_{B} T_{0}+\Delta_{\Gamma_{6}}}}{1+\sum_{\Gamma^{\prime}} \frac{2\left|V_{\Gamma^{\prime}}\right|^{2} N(0)}{k_{B} T_{0}+\Delta_{\Gamma^{\prime}}}} \sim \frac{\left|V_{\Gamma_{6}}\right|^{2}}{\left|V_{\Gamma_{7}^{(1)}}\right|^{2}} \frac{k_{B} T_{0}}{\Delta_{\Gamma_{6}}} \sim \frac{k_{B} T_{0}}{\Delta_{\Gamma_{6}}}
$$

since the hybridization strengths are comparable (see Fig. 5, renormalized quasiparticle bands). Taking $T_{0}=T_{K} \approx 20 \mathrm{~K}$ and $\Delta_{\Gamma_{6}} \approx 30 \mathrm{meV}$, we find about $6 \% \Gamma_{6}$ contribution. Similarly, with $\Delta_{\Gamma_{7}^{(2)}} \approx 30 \mathrm{meV}$, we may also expect to find about $6 \%$ of $\Gamma_{7}^{(2)}$ contribution in the ground state. These are small amounts but not negligible, and perhaps sufficient to justify the multiorbital model [19] that explains the symmetry of the superconducting gap at very low temperatures. 
The small but non-negligible amount of hybridizationinduced mixing of the excited states into the ground state is also very much supported by the results of renormalized band-structure calculations $[49,50]$. Here the present groundstate symmetry and excited $4 f$ states at $30 \mathrm{meV}$ are taken into account and the contributions of the three crystal-field states are projected out to the respective bands (see Fig. 5). The Fermi surface has two major sheets with quasiparticle masses that differ profoundly in which the Fermi surface sheet with the light quasiparticles very much resembles that of the LDA standard band-structure calculations. It indeed turns out that the heavy band at zero energy has mainly $\Gamma_{7}^{(1)}$ character with some contributions in the few percent range of the $\Gamma_{7}^{(2)}$ state and, due to a smaller hybridization function, somewhat less of the $\Gamma_{6}$.

Finally, for completeness, we need to examine the consequences of having a mixed-orbital ground state for the analysis of the linear dichroism in XAS. We calculated the XAS spectra for a ground state which consists of $88 \% \Gamma_{7}^{(1)}$, $6 \%$ of $\Gamma_{6}$, and $6 \%$ of $\Gamma_{7}^{(2)}$ states. This requires the adjustment of the $\alpha$ value to $|0.62|$ in order to reproduce the experiment. The correction is minor not only because the contribution of the higher lying states is small, but also because the linear dichroism of the $\Gamma_{6}$ and $\Gamma_{7}^{(2)}$ states partially cancel each other out. The small correction to $\alpha$ makes us confident that the crystal-field model with the Boltzmann occupation of the excited states is of more than sufficient accuracy to support our conclusion that no orbital switching as function of temperature takes place. Instead, we can safely infer the presence of a robust static crystal-field scheme, although, in principle, the analysis of the temperature dependence of the linear dichroism would require a temperature-dependent Anderson impurity calculation. It would also be very interesting to study quantitatively in the same framework the impact of the mixing of the $\Gamma_{6}$ and $\Gamma_{7}^{(2)}$ on the magnetic susceptibility.

The present data, the Anderson impurity, and LDA calculations principally allow for a multiorbital ground state that includes the $\Gamma_{6}$ crystal-field state that is expected for the $d+d$ singlet pairing state as proposed by $\mathrm{Nica}$ and $\mathrm{Si}$. Their analysis involves $\Gamma_{6}$ Wannier orbitals of the conduction electron states near the Fermi energy. These Wannier orbitals will hybridize with the excited $\Gamma_{6}$ crystal-field state of the $f$ manifold and thereby make it a small but nonzero component in the ground state $[19,51]$.

\section{SUMMARY}

$\mathrm{CeCu}_{2} \mathrm{Si}_{2}$ has been investigated with soft $\mathrm{x}$-ray absorption spectroscopy in the temperature range from $250 \mathrm{mK}$ to $250 \mathrm{~K}$ and the $J_{z}$ admixture of the $\Gamma_{7}$ ground-state wave function has been determined. The overall temperature dependence of the experimental linear dichroism is well reproduced by the thermal occupation of excited crystal-field states so that the scenario of orbital switching seems unlikely. The spectra indicate the presence of the $\mathrm{Ce} 4 f^{0}$ configuration in the ground state so that in principle the ground state can have a multiorbital character. Based on the experimentally confirmed Kondo temperature and the crystal-field energies, the contribution of the higher lying $\Gamma_{6}$ and $\Gamma_{7}^{(2)}$ crystal-field states to the primarily $\Gamma_{7}^{(1)}$ ground state is estimated to be about $6 \%$ within the $4 f^{1}$ manifold. This estimate is supported by renormalized bandstructure calculations that uses the experimentally determined crystal-field scheme.

\section{ACKNOWLEDGMENTS}

We would like to thank P. Thalmeier for various discussion and input. We further acknowledge Synchrotron-SOLEIL and synchrotron-ALBA (Exp. Id. No. 2019023540) for provision of synchrotron radiation facilities and the support from the Max Planck-POSTECH-Hsinchu Center for Complex Phase Materials. This work has benefited from funding from LabEx PALM (ANR-10-LABX-0039-PALM), and M.S. and A.S. gratefully acknowledge the financial support of the German funding agency Deutsche Forschungsgemeinschaft (DFG) through Grants No. SE 1441-4-1 and No. SE 1441-5-1. G.Z. acknowledges financial support through the ANR-DFG project Fermi-NESt (Deutsche Forschungsgemeinschaft Grant No. ZW 77/5-1).
[1] P. Coleman, in Handbook of Magnetism and Advanced Magnetic Materials, edited by M. F. S. M. H. Kronmüller, S. Parkin and I. Zutic (John Wiley and Sons, New York, 2007), Vol. 1, pp. 95-148.

[2] P. Coleman, in Many-Body Physics: From Kondo to Hubbard, edited by E. Pavarini, E. Koch, and P. Coleman (Schriften des Forschungszentrums Jülich, 2015), p. 95.

[3] H. v. Löhneysen, A. Rosch, M. Vojta, and P. Wölfle, Fermiliquid instabilities at magnetic quantum phase transitions, Rev. Mod. Phys. 79, 1015 (2007).

[4] S. Wirth and F. Steglich, Exploring heavy fermions from macroscopic to microscopic length scales, Nat. Rev. Mater. 1, 16066 (2016).

[5] P. Sun and F. Steglich, Nernst Effect: Evidence of Local Kondo Scattering in Heavy Fermions, Phys. Rev. Lett. 110, 216408 (2013).
[6] F. Steglich and S. Wirth, Foundations of heavy-fermion superconductivity: Lattice Kondo effect and Mott physics, Rep. Prog. Phys. 79, 084502 (2016).

[7] F. Steglich, J. Aarts, C. D. Bredl, W. Lieke, D. Meschede, W. Franz, and H. Schäfer, Superconductivity in the Presence of Strong Pauli Paramagnetism: $\mathrm{CeCu}_{2} \mathrm{Si}_{2}$, Phys. Rev. Lett. 43, 1892 (1979).

[8] F. Thomas, J. Thomasson, C. Ayache, C. Geibel, and F. Steglich, Precise determination of the pressure dependence of $T_{c}$ in the heavy-fermion superconductor $\mathrm{CeCu}_{2} \mathrm{Si}_{2}$, Phys. B (Amsterdam, Neth.) 186-188, 303 (1993).

[9] H. Q. Yuan, F. M. Grosche, M. Deppe, C. Geibel, G. Sparn, and F. Steglich, Observation of two distinct superconducting phases in $\mathrm{CeCu}_{2} \mathrm{Si}_{2}$, Science 302, 2104 (2003).

[10] O. Stockert, J. Arndt, E. Faulhaber, C. Geibel, H. S. Jeevan, S. Kirchner, M. Loewenhaupt, K. Schmalzl, W. Schmidt, Q. 
$\mathrm{Si}$, and F. Steglich, Magnetically driven superconductivity in $\mathrm{CeCu}_{2} \mathrm{Si}_{2}$, Nat. Phys. 7, 119 (2010).

[11] I. Eremin, G. Zwicknagl, P. Thalmeier, and P. Fulde, Feedback Spin Resonance in Superconducting $\mathrm{CeCu}_{2} \mathrm{Si}_{2}$ and $\mathrm{CeCoIn}_{5}$, Phys. Rev. Lett. 101, 187001 (2008).

[12] O. Stockert and F. Steglich, Unconventional quantum criticality in heavy-fermion compounds, Annu. Rev. Condens. Matter Phys. 2, 79 (2011).

[13] G. Pang, M. Smidman, J. Zhang, L. Jiao, Z. Weng, E. M. Nica, Y. Chen, W. Jiang, Y. Zhang, W. Xie, H. S. Jeevan, H. Lee, P. Gegenwart, F. Steglich, Q. Si, and H. Yuan, Fully gapped $d$-wave superconductivity in $\mathrm{CeCu}_{2} \mathrm{Si}_{2}$, Proc. Nat. Acad. Sci. USA 115, 5343 (2018).

[14] A. T. Holmes, D. Jaccard, and K. Miyake, Valence instability and superconductivity in heavy fermion systems, J. Phys. Soc. Jpn. 76, 051002 (2007).

[15] J.-P. Rueff, S. Raymond, M. Taguchi, M. Sikora, J.-P. Itié, F. Baudelet, D. Braithwaite, G. Knebel, and D. Jaccard, PressureInduced Valence Crossover in Superconducting $\mathrm{CeCu}_{2} \mathrm{Si}_{2}$, Phys. Rev. Lett. 106, 186405 (2011).

[16] S. Kittaka, Y. Aoki, Y. Shimura, T. Sakakibara, S. Seiro, C. Geibel, F. Steglich, H. Ikeda, and K. Machida, Multiband Superconductivity with Unexpected Deficiency of Nodal Quasiparticles in $\mathrm{CeCu}_{2} \mathrm{Si}_{2}$, Phys. Rev. Lett. 112, 067002 (2014).

[17] T. Yamashita, T. Takenaka, Y. Tokiwa, J. A. Wilcox, Y. Mizukami, D. Terazawa, Y. Kasahara, S. Kittaka, T. Sakakibara, M. Konczykowski, S. Seiro, H. S. Jeevan, C. Geibel, C. Putzke, T. Onishi, H. Ikeda, A. Carrington, T. Shibauchi, and Y. Matsuda, Fully gapped superconductivity with no sign change in the prototypical heavy-fermion $\mathrm{CeCu}_{2} \mathrm{Si}_{2}$, Sci. Adv. 3, e1601667 (2017).

[18] T. Takenaka, Y. Mizukami, J. A. Wilcox, M. Konczykowski, S. Seiro, C. Geibel, Y. Tokiwa, Y. Kasahara, C. Putzke, Y. Matsuda, A. Carrington, and T. Shibauchi, Full-Gap Superconductivity Robust Against Disorder in Heavy-Fermion $\mathrm{CeCu}_{2}$ $\mathrm{Si}_{2}$, Phys. Rev. Lett. 119, 077001 (2017).

[19] E. M. Nica and Q. Si, Multiorbital singlet pairing and $d+d$ superconductivity, arXiv:1911.13274.

[20] L. V. Pourovskii, P. Hansmann, M. Ferrero, and A. Georges, Theoretical Prediction and Spectroscopic Fingerprints of an Orbital Transition in $\mathrm{CeCu}_{2} \mathrm{Si}_{2}$, Phys. Rev. Lett. 112, 106407 (2014).

[21] E. A. Goremychkin and R. Osborn, Crystal-field excitations in $\mathrm{CeCu}_{2} \mathrm{Si}_{2}$, Phys. Rev. B 47, 14280 (1993). Note: on page $14287 \eta$ and $\sqrt{\left(1-\eta^{2}\right)}$ are exchanged. The correct notation is $\mid$ g.s. $\rangle=\sqrt{\left(1-\eta^{2}\right)}| \pm 5 / 2\rangle \pm \eta|\mp 3 / 2\rangle$ with $\sqrt{\left(1-\eta^{2}\right)}=0.88$ to reproduce the anisotropy of the static susceptibility.

[22] M. W. Haverkort, A. Tanaka, L. H. Tjeng, and G. A. Sawatzky, Nonresonant Inelastic X-Ray Scattering Involving Excitonic Excitations: The Examples of $\mathrm{NiO}$ and $\mathrm{CoO}$, Phys. Rev. Lett. 99, 257401 (2007).

[23] R. A. Gordon, G. T. Seidler, T. T. Fister, M. W. Haverkort, G. A. Sawatzky, A. Tanaka, and T. K. Sham, High multipole transitions in NIXS: Valence and hybridization in $4 f$ systems, EPL 81, 26004 (2008).

[24] R. A. Gordon, M. W. Haverkort, Subhra Sen Gupta, and G. A. Sawatzky, Orientation-dependent x-ray Raman scattering from cubic crystals: Natural linear dichroism in $\mathrm{MnO}$ and $\mathrm{CeO}_{2}$, J. Phys.: Conf. Ser. 190, 012047 (2009).
[25] T. Willers, F. Strigari, N. Hiraoka, Y. Q. Cai, M. W. Haverkort, K.-D. Tsuei, Y. F. Liao, S. Seiro, C. Geibel, F. Steglich, L. H. Tjeng, and A. Severing, Determining the In-Plane Orientation of the Ground-State Orbital of $\mathrm{CeCu}_{2} \mathrm{Si}_{2}$, Phys. Rev. Lett. 109, 046401 (2012). Note: Fig. 1 of Ref. [25] used $|\alpha|=0.88$ as proposed by Goremychkin, while Fig. 1 in this work uses the $|\alpha|$ value as determined in the present XAS experiment.

[26] A. Tanaka and T. Jo, Resonant $3 d, 3 p$, and $3 s$ photoemission in transition metal oxides predicted at $2 p$ threshold, J. Phys. Soc. Jpn. 63, 2788 (1994).

[27] Theo Thole Memorial Issue, J. Electron Spectroscopy Relat. Phenom. 86(1-3) (1997).

[28] F. de Groot and A. Kotani, Core Level Spectroscopy of Solids (Taylor and Francis Group, Philadelphia, 2008).

[29] P. Hansmann, A. Severing, Z. Hu, M. W. Haverkort, C. F. Chang, S. Klein, A. Tanaka, H. H. Hsieh, H.-J. Lin, C. T. Chen, B. Fåk, P. Lejay, and L. H. Tjeng, Determining the Crystal-Field Ground State in Rare Earth Heavy Fermion Materials Using Soft-X-Ray Absorption Spectroscopy, Phys. Rev. Lett. 100, 066405 (2008).

[30] T. Willers, Z. Hu, N. Hollmann, P. O. Körner, J. Gegner, T. Burnus, H. Fujiwara, A. Tanaka, D. Schmitz, H. H. Hsieh, H.-J. Lin, C. T. Chen, E. D. Bauer, J. L. Sarrao, E. Goremychkin, M. Koza, L. H. Tjeng, and A. Severing, Crystal-field and kondo-scale investigations of $\mathrm{Ce} M \operatorname{In}_{5}(M=\mathrm{Co}$, Ir, and $\mathrm{Rh})$ : A combined $\mathrm{x}$-ray absorption and inelastic neutron scattering study, Phys. Rev. B 81, 195114 (2010).

[31] T. Willers, D. T. Adroja, B. D. Rainford, Z. Hu, N. Hollmann, P. O. Körner, Y.-Y. Chin, D. Schmitz, H. H. Hsieh, H.-J. Lin, C. T. Chen, E. D. Bauer, J. L. Sarrao, K. J. McClellan, D. Byler, C. Geibel, F. Steglich, H. Aoki, P. Lejay, A. Tanaka, L. H. Tjeng, and A. Severing, Spectroscopic determination of crystal-field levels in $\mathrm{CeRh}_{2} \mathrm{Si}_{2}$ and $\mathrm{CeRu}_{2} \mathrm{Si}_{2}$ and of the $4 f^{0}$ contributions in $\mathrm{Ce}_{2} \mathrm{Si}_{2}(M=\mathrm{Cu}, \mathrm{Ru}, \mathrm{Rh}, \mathrm{Pd}$, and $\mathrm{Au})$, Phys. Rev. B 85, 035117 (2012).

[32] F. Strigari, T. Willers, Y. Muro, K. Yutani, T. Takabatake, Z. Hu, Y.-Y. Chin, S. Agrestini, H.-J. Lin, C. T. Chen, A. Tanaka, M. W. Haverkort, L. H. Tjeng, and A. Severing, Crystal-field ground state of the orthorhombic Kondo insulator $\mathrm{CeRu}_{2} \mathrm{Al}_{10}$, Phys. Rev. B 86, 081105(R) (2012).

[33] T. Willers, F. Strigari, Z. Hu, V. Sessi, N. Brookes, E. Bauer, J. Sarrao, J. Thompson, A. Tanaka, S. Wirth, L. Tjeng, and A. Severing, Correlation between ground state and orbital anisotropy in heavy fermion materials, Proc. Nat. Acad. Sci. USA 112, 2384 (2015).

[34] O. Gunnarsson and K. Schönhammer, Electron spectroscopies for ce compounds in the impurity model, Phys. Rev. B 28, 4315 (1983).

[35] S. Seiro, M. Deppe, H. Jeevan, U. Burkhardt, and C. Geibel, Flux crystal growth of $\mathrm{CeCu}_{2} \mathrm{Si}_{2}$ : Revealing the effect of composition, Phys. Status Solidi B 247, 614 (2010).

[36] L. Joly, E. Otero, F. Choueikani, F. Marteau, L. Chapuis, and P. Ohresser, Fast continuous energy scan with dynamic coupling of the monochromator and undulator at the DEIMOS beamline, J. Synchrotron Radiat. 21, 502 (2014).

[37] A. Barla, J. Nicolas, D. Cocco, S. Valvidares, J. Herrero-Martin, P. Gargiani, J. Moldes, C. Ruget, E. Pellegrin, and S. Ferrer, Design and performance of BOREAS, the beamline for resonant $\mathrm{x}$-ray absorption and scattering experiments at the ALBA 
synchrotron light source, J. Synchrotron Radiat. 23, 1507 (2016).

[38] J.-P. Kappler, E. Otero, W. Li, L. Joly, G. Schmerber, B. Muller, F. Scheurer, F. Leduc, B. Gobaut, L. Poggini, G. Serrano, F. Choueikani, E. Lhotel, A. Cornia, R. Sessoli, M. Mannini, M.-A. Arrio, P. Sainctavit, and P. Ohresser, Ultralow-temperature device dedicated to soft x-ray magnetic circular dichroism experiments, J. Synchrotron Radiat. 25, 1727 (2018).

[39] M. W. Haverkort, QUANTY for core level spectroscopy: Excitons, resonances, and band excitations in time and frequency domain, J. Phys.: Conf. Ser. 712, 012001 (2016).

[40] R. Cowan, The Theory of Atomic Structure and Spectra (University of California Press, San Francisco, 1981).

[41] S. Horn, E. Holland-Moritz, M. Loewenhaupt, F. Steglich, H. Scheuer, A. Benoit, and J. Flouquet, Magnetic neutron scattering and crystal-field states in $\mathrm{CeCu}_{2} \mathrm{Si}_{2}$, Phys. Rev. B 23, 3171 (1981).

[42] J.-P. Rueff, J. M. Ablett, F. Strigari, M. Deppe, M. W. Haverkort, L. H. Tjeng, and A. Severing, Absence of orbital rotation in superconducting $\mathrm{CeCu}_{2} \mathrm{Ge}_{2}$, Phys. Rev. B 91, 201108(R) (2015).

[43] M. Sundermann, F. Strigari, T. Willers, H. Winkler, Prokofiev, J. M. A. Ablett, J.-P. Rueff, D. Schmitz, E. Weschke, M. Sala-Moretti, A. Al-Zein, A. Tanaka, M. W. Haverkort, D. Kasinathan, L. H. Tjeng, S. Paschen, and A. Severing, $\mathrm{CeRu}_{4} \mathrm{Sn}_{6}$ : A strongly correlated material with nontrivial topology, Sci. Rep. 5, 17937 (2015).
[44] M. Sundermann, A. Amorese, F. Strigari, B. Leedahl, L. H. Tjeng, M. W. Haverkort, H. Gretarsson, H. Yavaş, M. Moretti Sala, E. D. Bauer, P. F. S. Rosa, J. D. Thompson, and A. Severing, Orientation of the ground-state orbital in $\mathrm{CeCoIn}_{5}$ and CeRhIn $_{5}$, Phys. Rev. B 99, 235143 (2019).

[45] N. E. Bickers, D. L. Cox, and J. W. Wilkins, Self-consistent large- $N$ expansion for normal-state properties of dilute magnetic alloys, Phys. Rev. B 36, 2036 (1987).

[46] K. Kummer, C. Geibel, C. Krellner, G. Zwicknagl, C. Laubschat, N. B. Brookes, and D. V. Vyalikh, Similar temperature scale for valence changes in Kondo lattices with different Kondo temperatures, Nat. Commun. 9, 2011 (2018).

[47] G. Zwicknagl, V. Zevin, and P. Fulde, Simple approximation scheme for the Anderson impurity Hamiltonian, Z. Phys. B 79, 365 (2010).

[48] C. M. Varma and Y. Yafet, Magnetic susceptibility of mixedvalence rare-earth compounds, Phys. Rev. B 13, 2950 (1976).

[49] G. Zwicknagl, Quasi-particles in heavy fermion systems, Adv. Phys. 41, 203 (1992)

[50] G. Zwicknagl and U. Pulst, $\mathrm{CeCu}_{2} \mathrm{Si}_{2}$ : Renormalized band structure, quasiparticles, and co-operative phenomena, J. Phys. B 186-188, 895 (1993).

[51] Q. Si (private communication).

Correction: The surname of the seventh author contained an error and has been fixed. 